\title{
Malaria transmission and vector behaviour in a forested malaria focus in central Vietnam and the implications for vector control
}

\author{
Wim Van Bortel ${ }^{1 *}$, Ho Dinh Trung ${ }^{2}$, Le Xuan Hoi ${ }^{2}$, Nguyen Van Ham³ ${ }^{3}$ Nguyen Van Chut ${ }^{3}$, Nguyen Dinh Luu ${ }^{2}$, \\ Patricia Roelants ${ }^{1}$, Leen Denis ${ }^{1}$, Niko Speybroeck ${ }^{4,5}$, Umberto D'Alessandro $^{1}$, Marc Coosemans ${ }^{1,6}$
}

\begin{abstract}
Background: In Vietnam, malaria is becoming progressively restricted to specific foci where human and vector characteristics alter the known malaria epidemiology, urging for alternative or adapted control strategies. Longlasting insecticidal hammocks (LLIH) were designed and introduced in Ninh Thuan province, south-central Vietnam, to control malaria in the specific context of forest malaria. An entomological study in this specific forested environment was conducted to assess the behavioural patterns of forest and village vectors and to assess the spatio-temporal risk factors of malaria transmission in the province.

Methods: Five entomological surveys were conducted in three villages in Ma Noi commune and in five villages in Phuoc Binh commune in Ninh Thuan Province, south-central Vietnam. Collections were made inside the village, at the plot near the slash-and-burn fields in the forest and on the way to the forest. All collected mosquito species were subjected to enzyme-linked immunosorbent assay (ELISA) to detect Plasmodium in the head-thoracic portion of individual mosquitoes after morphological identification. Collection data were analysed by use of correspondence and multivariate analyses.
\end{abstract}

Results: The mosquito density in the study area was low with on average 3.7 anopheline bites per man-night and 17.4 culicine bites per man-night. Plasmodium-infected mosquitoes were only found in the forest and on the way to the forest. Malaria transmission in the forested malaria foci was spread over the entire night, from dusk to dawn, but was most intense in the early evening as nine of the 13 Plasmodium positive bites occurred before $21 \mathrm{H}$. The annual entomological inoculation rate of Plasmodium falciparum was 2.2 infective bites per person-year to which Anopheles dirus s.s. and Anopheles minimus s.s. contributed. The Plasmodium vivax annual entomological inoculation rate was 2.5 infective bites per person-year with Anopheles sawadwongporni, Anopheles dirus s.s. and Anopheles pampanai as vectors.

Conclusion: The vector behaviour and spatio-temporal patterns of malaria transmission in Southeast Asia impose new challenges when changing objectives from control to elimination of malaria and make it necessary to focus not only on the known main vector species. Moreover, effective tools to prevent malaria transmission in the early evening and in the early morning, when the treated bed net cannot be used, need to be developed.

\section{Background}

Long-lasting insecticidal materials are central in the prevention and control of malaria. To be successful they need to be adapted to the local epidemiological context. Vietnam has demonstrated that a substantial reduction of

\footnotetext{
* Correspondence: wvbortel@itg.be

${ }^{1}$ Institute of Tropical Medicine Antwerp, Dept. Parasitology, Nationalestraat 155, B-2000 Antwerpen, Belgium

Full list of author information is available at the end of the article
}

malaria-related mortality and morbidity is feasible. Political commitment to malaria control and internal and external funding were key to this success $[1,2]$. However, malaria elimination in Vietnam can only be achieved if the country is able to cope with the remaining malaria endemic foci. These foci are situated in the central highlands and along international borders of Laos and Cambodia where the majority of the severe cases and malaria-related deaths occurs [3]. These areas are remote, forested and

\section{Ciomed Central}


populated by ethnic minorities living traditionally on forest related activities. These activities have been identified as a strong risk factor for malaria infection $[4,5]$. The vector present in this forested area, Anopheles dirus, is exophagic and exophilic, jeopardizing the impact of the traditional control measures [6]. To protect this specific human population, an adapted preventive measure, namely longlasting insecticidal hammocks (LLIH), were proposed. LLIH were designed to control malaria in the specific forest context where people use hammocks. It consisted of a green nylon hammock to which Olyset ${ }^{\oplus}$ netting was stitched. The netting was double the width of the hammock itself [7]. Half was sewn onto the back of the hammock while the other half served as a free flap to cover the person inside. A community based-cluster randomized intervention, assessing the impact of the LLIH, was implemented in the province of Ninh Thuan Vietnam and showed that LLIH reduced malaria incidence and prevalence, especially in the cluster with the highest malaria burden [8]. Knowledge of the behaviour of humans [9] and vectors are important for the success of this control measure. Therefore, an entomological study was conducted, in the framework of the above mentioned community-based trial, to assess the behavioural patterns of forest and village vectors and to assess the spatio-temporal risk factors of malaria transmission in this province.

\section{Methods}

\section{Study place}

The study was conducted in the province of Ninh Thuan in south-central Vietnam. The province has about 570,000 inhabitants and is divided into six districts. The Ra-glai people of Ninh Thuan combine living in villages along the road with a second home at their slash and burn fields in the forest. In the forest, houses are generally built on stilts whereas in the villages two types of houses, built on the ground or on stilts, are present. They depend on the forest for their subsistence where they are at high risk of getting malaria. They grow maize, cashew nuts, rice, beans and manioc, cultivate cash crops such as coffee and cotton, and exploit forest products, such as bamboo, resin, and hunting. The dry season in this province lasts from January to April, the rainy season from May till December. The mean annual rainfall is $725 \mathrm{~mm}$ per year with the mean temperature ranging from 25 to $30^{\circ} \mathrm{C}$ and the humidity between 70 and $80 \%$. Malaria transmission shows a peak in May-June at the start of the rainy season and in October-November at the end of the rainy season.

\section{Mosquito collections}

Five entomological surveys (November 2004, October and November 2005, October and November 2006) were conducted in three villages in Ma Noi commune and in five villages in Phuoc Binh commune. Collections were made in two different places per study village i.e. inside the village (subsequently called village) and at the plot near the slash and burn fields in the forest (subsequently called forest). In one village per commune collections were made in three different places on a road frequented by the inhabitants to go from the village to the forest plot (subsequently called 'way'). In each collection place, outdoor human landing collections were made during eight nights per survey. In the village, two houses were selected, each with one collector outdoors, in the forest two places were selected with each time two collectors, and on the 'way' each site was sampled by two collectors. From survey 3 , indoor human collections were made inside the village in two houses by one collector to assess the indoor and outdoor mosquito density in the village. In the village and forest sites, collections were made from $18 \mathrm{H}$ till $06 \mathrm{H}$ by two teams. A first team worked from $18 \mathrm{H}$ to $24 \mathrm{H}$ and a second team from $24 \mathrm{H}$ to $6 \mathrm{H}$. On the way from village to forest collections were made from $17 \mathrm{H}$ till $20 \mathrm{H}$ and from $04 \mathrm{H}$ till $07 \mathrm{H}$.

Mosquitoes were stored by collection hour and morphologically identified in the field by use of a standardized key for medically important anophelines of Southeast Asia [10]. Mosquitoes were individually stored in small tubes over silica gel for subsequent analyses.

\section{Laboratory analyses}

All collected mosquito species were subjected to enzyme-linked immunosorbent assay (ELISA) to detect Plasmodium falciparum, Plasmodium vivax 210 and P. vivax 247 circumsporozoite protein (CSP) in the head-thoracic portion of individual mosquitoes [11-13]. Positive CSP ELISA mosquitoes were confirmed by PCR. DNA was extracted from the ELISA homogenate using the QIAamp DNA micro kit (Qiagen) following the manufacturer's instructions. Amplification of Plasmodium was done by use of the primers PL1473F18 and PL1679R18 targeting the 18SrRNA [14], amplicons were subsequently cloned using the Original TA cloning kit according the manufacturer's instructions (Invitrogen, Carlsbad, California) and sequenced (GenoScreen, Lille, France). Sequences were used to confirm the Plasmodium species identification based on ELISA.

The morphological identification of the mosquitoes found positive for ELISA was confirmed by PCR using the PCR-RFLP for Anopheles minimus complex and Anopheles pampanai [15], and the allele specific PCR for An. dirus complex [16]. The identification of Anopheles maculatus s.l. was confirmed by sequencing (GenoScreen, Lille, France) the ITS2 rDNA region using primers ITS2A and ITS2B described in Beebe \& Saul [17]. The sequences were blasted and compared with reference sequences described in Walton et al [18]. 


\section{Statistical analysis}

The association between the Anopheles species with collection places (communes and forest/village combined) was evaluated by applying correspondence analysis (CA) on the contingency table of the collection data. CA is a multivariate technique most similar to factor analysis, but applicable on a two-way contingency table of observed frequencies. Chi-square distances provide a standardized measure of association between the rows and columns of the contingency table. CA transforms these association measures into metric distance measure and creates orthogonal dimensions upon which the categories can be placed to best account for the strength of association represented by the chi-square distances [19]. The degree of association or correspondence is reflected by the metric distances in the ordination plot, so that the smaller the distances between points, the "stronger" their association [19]. CA was performed using Stata 10.0 (Stata Corp. College Station, TX).

Differences in Anopheles densities (bites per mannight) were tested by a negative binomial regression taking into account the sampling design in which survey and village name were the clustering variables (command survey set in Stata). Collection place (village and forest), commune (Ma Noi, Phuoc Binh) and intervention were the explanatory variables. The study was not designed to assess the impact of the intervention on the mosquito density. Yet the LLIH intervention started in November 2004 and to account for the possible effect of intervention on the density it was included as explanatory variable in the analysis.

\section{Ethical consideration}

The study protocol was approved by the ethical committees of NIMPE, Hanoi, Vietnam and of the Institute of Tropical Medicine, Antwerp, Belgium as well as by the Vietnamese Ministry of Health. The mosquito collectors and householders were informed about the objectives, process and procedures of the study and oral informed consent was sought from them. Collector candidates were invited among the adult village population and if individuals wished to withdraw, they were allowed to do so at any time without prejudice. Access to malaria diagnosis and treatment was guaranteed throughout the study.

\section{Results}

\section{The spatial contact with the Anopheles species}

In total, 24 different Anopheles species were collected outdoors in the village and forest. These collections counted 1821 person-nights divided over five surveys. Survey one counted 288 person-nights, surveys two, three and five 383 person-nights and survey four 384 person nights. During the first survey sampling was restricted to three villages. Following species were only sporadically collected i.e. less than 10 specimens for each of these species were found during the entire study: Anopheles annandalei, Anopheles argyropus, Anopheles barbumbrosus, Anopheles crawfordi, Anopheles jamesii, Anopheles jeyporiensis, Anopheles monstrosus, Anopheles tessellatus, Anopheles vagus and Anopheles varuna. These mosquitoes were not further considered in the analysis of the biting rates. The 14 remaining species were collected in both the forest and the village sites though at different densities resulting in a significant association between species, collection place and communes (Figure 1, Table 1). Anopheles dirus s.s., the only species of the complex occurring in this area [20], and An. maculatus s.l. were significantly associated with the forest in both Phuoc Binh and Ma Noi, while $A n$. minimus s.l. was identified as a forest species associated with Ma Noi commune (Figure 1, Table 1). Anopheles pampanai was only collected in the commune of Ma Noi and showed an association with the forest (Figure 1). This trend was also observed in the regression analysis though the difference in density between forest and village was not significant for this species (Table 1). Anopheles aconitus, Anopheles annularis, Anopheles nigerrimus, Anopheles philippinensis and Anopheles sinensis were significantly more abundant in the villages. The village sites in Phuoc Binh were more similar in terms of species composition to the forest places of Ma Noi and Phuoc Binh than to the village sites in Ma Noi (Figure 1).

Overall the species density in the study area was low with on average 3.7 anopheline bites per man-night and 17.4 culicine bites per man-night $(\mathrm{BMN})$. The mean outdoor Anopheles density in the forest was 4.3 BMN and in the village $2.5 \mathrm{BMN}$ ( $\mathrm{p}=0.007)$. In the village the Anopheles outdoor biting density (2.5 BMN) was significantly higher than the indoor biting density $(0.7$ $\mathrm{BMN})(\mathrm{p}=0.004)$. A similar pattern in the village was observed for $A n$. dirus (0.12 BMN outdoors versus 0.02 BMN indoors, $\mathrm{p}=0.035)$, An. maculatus s.l. (1.47 BMN outdoors versus $0.42 \mathrm{BMN}$ indoors, $\mathrm{p}<0.01$ ) and $A n$. minimus s.l. (0.07 BMN outdoors versus $0.008 \mathrm{BMN}$ indoors, $\mathrm{p}=0.023)$.

\section{The temporal contact with the Anopheles species}

The Anopheles species in the study area were early biters and almost no difference in biting time was observed between the forest and the village. In the forest, people were exposed to $45 \%$ of the total number of Anopheles bites by $21 \mathrm{H}$ which is the normal sleeping time for Ra-glai in the forest setting. In the village, where people go to bed no later than $22 \mathrm{H}$, they were exposed to $64 \%$ of the Anopheles bites before going to sleep. The temporal exposure to the vector species, 

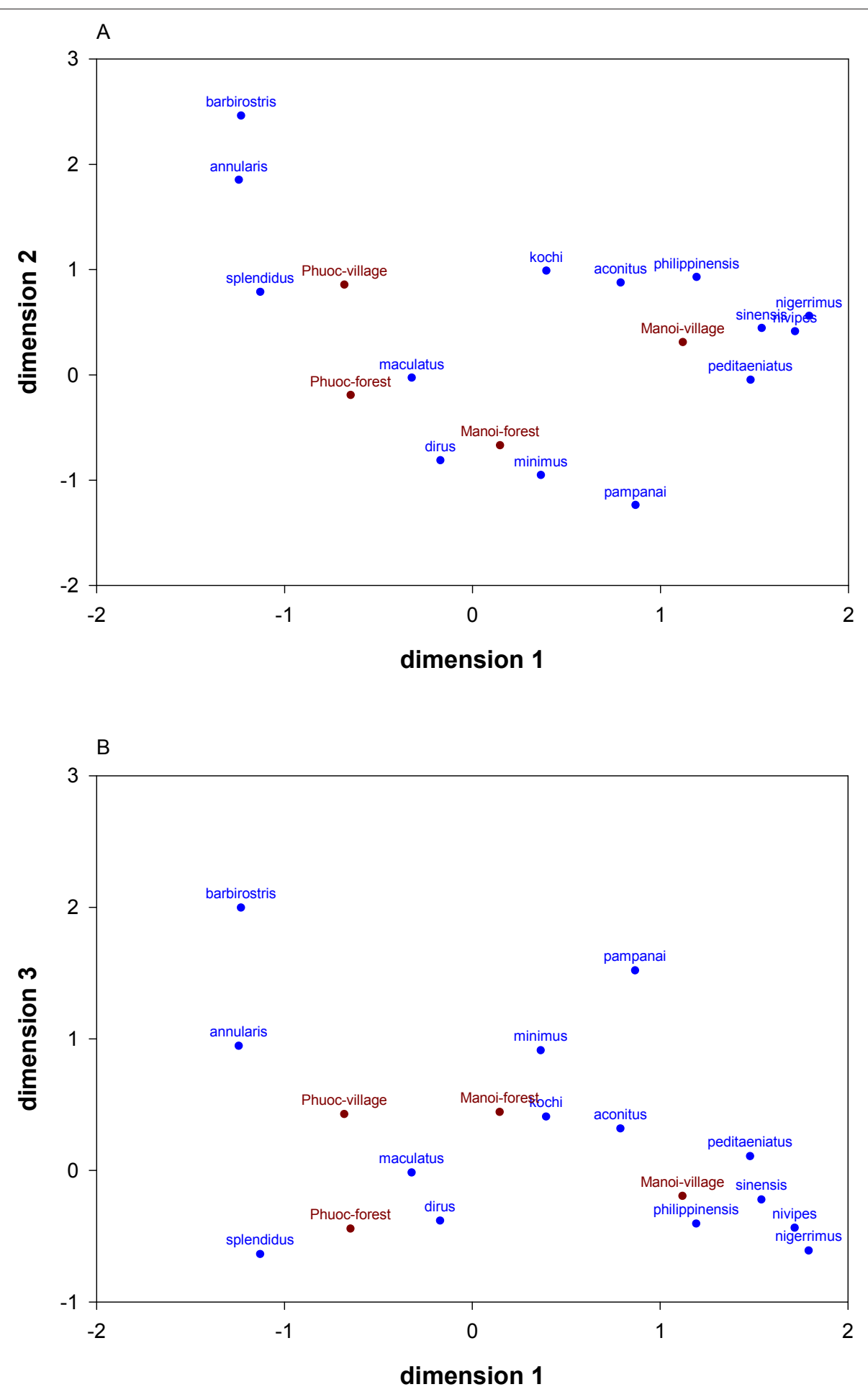

Figure 1 Two-dimensional ordinations (plots A and B) of the 3-dimensional correspondence analysis showing the collected Anopheles species (i.e. raw categories, blue) and the village-collection sites (i.e. column categories, red). The dimensions in the ordination plot show the metric distances. The smaller the distances between points, the "stronger" their associate i.e. points of a certain category positioned close to each other are similar with regard to the pattern of relative frequencies across the other category. Village-collection sites; Phuoc-village: village in Phuoc Binh, Phuoc-forest: forest in Phuoc Binh, Manoi-village: village in Ma Noi, Manoi-forest: forest in Ma Noi. 
Table 1 The mean density (bites per man-night) per Anopheles species by collection place (forest/village), Commune (Ma Noi/Phuoc Binh) and intervention. The significance levels were obtained from a multivariate binomial regression analysis including collection place, communes and intervention as explanatory variables.

\begin{tabular}{|c|c|c|c|c|c|c|c|c|c|c|c|c|}
\hline \multirow[b]{2}{*}{$\begin{array}{l}\text { Anopheles } \\
\text { species }\end{array}$} & \multicolumn{4}{|c|}{ Effect Forest/village } & \multicolumn{4}{|c|}{ Effect Commune } & \multicolumn{4}{|c|}{ Effect intervention } \\
\hline & Forest & & Village & p-value* & Ma Noi & & Phuoc Binh & p-value* & Control & & Inter-vention & p-value* \\
\hline aconitus & 0,059 & $<$ & 0,178 & 0,013 & 0,157 & & 0,061 & 0,143 & 0,143 & $>$ & 0,038 & 0,005 \\
\hline annularis & 0,022 & $<$ & 0,041 & 0,003 & 0 & $<$ & 0,047 & 0,000 & 0,000 & $<$ & 0,068 & 0,000 \\
\hline barbirostris & 0,012 & & 0,056 & 0,133 & 0,001 & & 0,043 & 0,061 & 0,031 & & 0,020 & 0,434 \\
\hline dirus & 0,539 & $>$ & 0,106 & 0,011 & 0,364 & & 0,415 & 0,972 & 0,355 & & 0,449 & 0,632 \\
\hline kochi & 0,009 & & 0,020 & 0,126 & 0,013 & & 0,013 & 0,846 & 0,009 & & 0,018 & 0,141 \\
\hline maculatus & 2,941 & $>$ & 1,356 & 0,005 & 1,831 & & 2,795 & 0,444 & 2,624 & & 2,123 & 0,428 \\
\hline minimus & 0,240 & $>$ & 0,092 & 0,001 & 0,332 & $>$ & 0,098 & 0,021 & 0,234 & & 0,131 & 0,743 \\
\hline nigerrimus & 0,030 & $<$ & 0,140 & 0,020 & 0,169 & $>$ & 0 & 0,000 & 0,114 & $>$ & 0,003 & 0,011 \\
\hline nivipes & 0,013 & & 0,035 & 0,052 & 0,051 & $>$ & 0 & 0,000 & 0,035 & $>$ & 0 & 0,000 \\
\hline pampanai & 0,026 & & 0,012 & 0,125 & 0,054 & $>$ & 0 & 0,000 & 0,022 & & 0,021 & 0,548 \\
\hline peditaeniatus & 0,122 & & 0,172 & 0,511 & 0,35 & $>$ & 0 & 0,000 & 0,173 & & 0,090 & 0,837 \\
\hline philippinensis & 0,003 & $<$ & 0,013 & 0,045 & 0,011 & & 0,004 & 0,348 & 0,009 & & 0,004 & 0,667 \\
\hline sinensis & 0,040 & $<$ & 0,102 & 0,000 & 0,141 & $>$ & 0,007 & 0,005 & 0,098 & $>$ & 0,008 & 0,046 \\
\hline splendidus & 0,229 & & 0,141 & 0,000 & 0,007 & $<$ & 0,326 & 0,000 & 0,294 & $>$ & 0,007 & 0,026 \\
\hline
\end{tabular}

* No adjustments were made to account for multiple testing. These corrections have been challenged [30] and the use of uncorrected p-values was preferred as indicative of an effect.

An. dirus, An. maculatus s.l and An. minimus s.l. was similar (Figure 2). These species were already active early in the night with up to $13 \%$ of the bites for $A n$. maculatus s.l. by $19 \mathrm{H}$. Likewise, this early activity was observed on the 'way' where the three species were collected before $18 \mathrm{H}$. At the early morning An. maculatus s.l. showed still some activity until $7 \mathrm{H}$ (Figure 3 ).

\section{Malaria transmission and the annual entomological inoculation rate}

Plasmodium infected mosquitoes were only found in the forest and on the 'way' (Table 2) in both communes Ma Noi and Phuoc Binh. Anopheles dirus s.s. and An. minimus s.s. were found infected with Plasmodium falciparum while Plasmodium vivax was detected in An. dirus s.s., An. maculatus s.l. (molecularly identified as Anopheles sawadwongporni), and An. pampanai. The risk of people to contact infected mosquitoes in their forest plot was high during their evening activities as nine of the 13 infected mosquitoes ( $P$. falciparum and $P$. vivax combined) were found by 21 hours (Figure 4). Plasmodium falciparum infected mosquitoes were also found in the forest in the second half of the night and on the 'way' two infective mosquitoes were observed in the early morning between 4-5 hours. The annual entomological inoculation rate of P. falciparum was 2.2 infective bites per person-year to which $A n$. dirus s.s. contributed $85 \%$ and $A n$. minimus s.s. $15 \%$. The $P$. vivax annual entomological inoculation rate was 2.5 infective bites per person-year to which $A n$. maculatus s.l. (molecularly identified as An. sawadwongporni) added $47 \%$, An. dirus s.s. $37 \%$ and $A n$. pampanai $16 \%$.

\section{Discussion}

In Ninh Thuan province, south central Vietnam, the biting nuisance of mosquitoes (Culicidae) in general and of Anopheles in particular was low both in the villages and in the forest, though the Anopheles density in the forest was significantly higher than in the village. All the Anopheles species considered in the analyses were found in both forest and village. Nevertheless, $A n$ dirus, $A n$. maculatus s.l. and An. minimus s.l. were more abundant

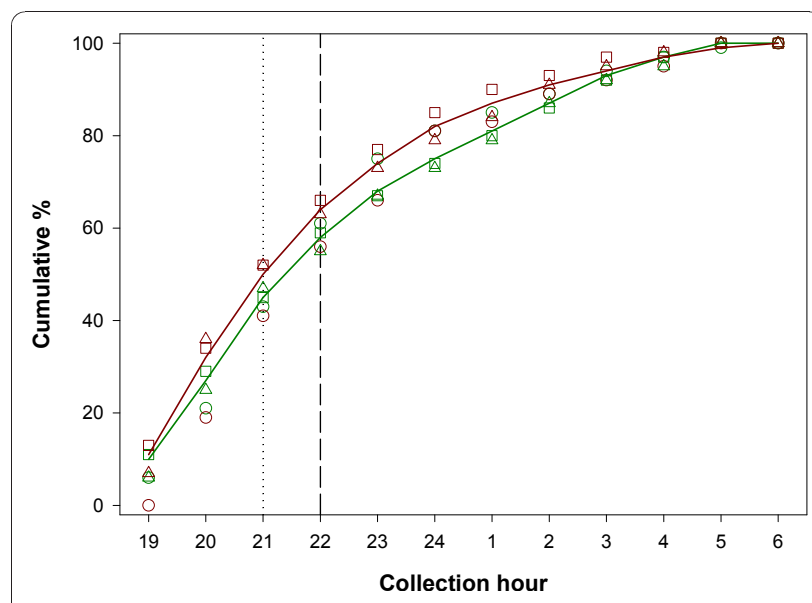

Figure 2 Cumulative biting rate of Anopheles species (straight line), Anopheles dirus (circles), Anopheles maculatus s.l. (triangles) and Anopheles minimus s.l. (squares) during the night. Green: collections in the forest, Red: collections in the village. The vertical dotted line indicates the human sleeping time in the forest. The vertical dashed line indicates the human sleeping time in the village [9]. 


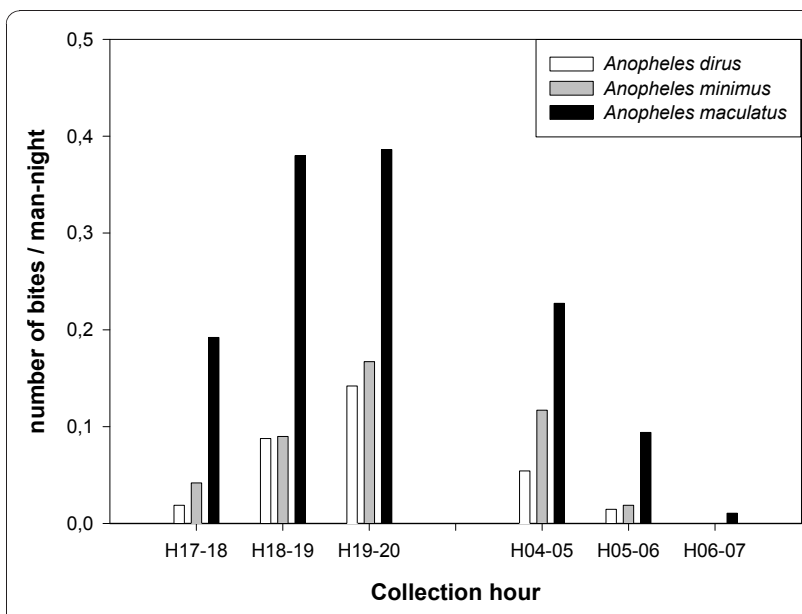

Figure 3 The biting activity of Anopheles dirus, Anopheles maculatus s.l. and Anopheles minimus s.l. on the 'way' between village and forest plot (all data pooled).

in the forest and had an early biting activity, corroborating previous observations [6].This study showed that these three mosquito species started their biting activity before $18 \mathrm{H}$ and that in the early morning An. maculatus s.l. was still active on the 'way'. Malaria transmission could only be detected in the forest and on the 'way'. The highest risk of contact with infected mosquitoes was during their evening activities. However, transmission also took place during the night and the early morning showing the importance to protect against mosquito bites from dusk to dawn. The annual entomological inoculation rate showed a level that was much

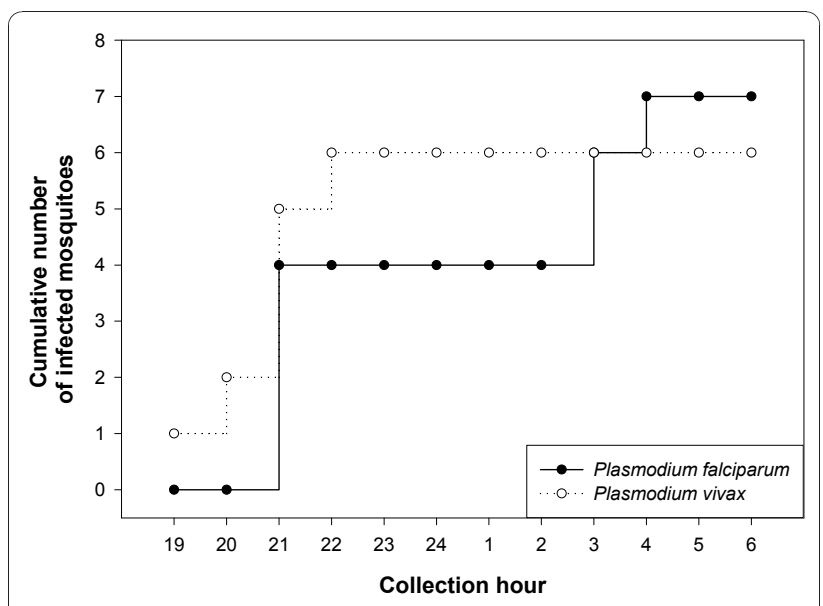

Figure 4 Cumulative number of infected Anopheles mosquitoes observed during the night in the forest (all data pooled).

lower than the one observed in Africa [21-23], but similar to what could be expected in malaria endemic foci in Southeast Asian mainland [24].

The Ra-glai people of Ninh Thuan combine living in villages along the road with a second home at their slash and burn fields in the forest. In these communities bed net (ITN) use in the villages was $85 \%$ but only $53 \%$ in the forest. About $20 \%$ slept unprotected in both places [9]. LLIH were introduced as a complementary control measure to protect these people against malaria transmission in the forest. The LLIH, utilized both indoors and outdoors, were more used during the day in both the villages and the forest $(69 \%$ and $73 \%$

Table 2 Overview of the Plasmodium infected mosquitoes as detected by ELISA and confirmed by PCR.

\begin{tabular}{|c|c|c|c|c|}
\hline \multirow[b]{2}{*}{ Collection place } & \multirow[b]{2}{*}{$\begin{array}{l}\text { Anopheles species } \\
\text { (morphological identification) }\end{array}$} & \multicolumn{3}{|c|}{ Number of Plasmodium sporozoite infected mosquitoes (number tested } \\
\hline & & P. falciparum & P. vivax 210 & P. vivax 247 \\
\hline \multirow[t]{4}{*}{ Forest } & An. dirus ${ }^{1}$ & $6(642)$ & $2(642)$ & $2(485)$ \\
\hline & An. maculatus s....$^{2}$ & $0(3454)$ & $0(3454)$ & $2(1826)$ \\
\hline & An. minimus s.l. ${ }^{3}$ & $1(264)$ & $0(264)$ & $0(166)$ \\
\hline & An. pampanai ${ }^{4}$ & $0(23)$ & $0(23)$ & $1(23)$ \\
\hline \multirow[t]{4}{*}{ Way } & An. dirus ${ }^{1}$ & $2(151)$ & $0(151)$ & $0(103)$ \\
\hline & An. maculatus s.l. & $0(618)$ & $0(618)$ & $0(524)$ \\
\hline & An. minimus s.l. & $0(204)$ & $0(204)$ & $0(144)$ \\
\hline & An. pampanai & $0(37)$ & $0(37)$ & $0(37)$ \\
\hline \multirow[t]{4}{*}{ Village } & An. dirus s.l. & $0(71)$ & $0(71)$ & $0(43)$ \\
\hline & An. maculatus s.l. & 0 (1009) & 0 (1009) & $0(650)$ \\
\hline & An. minimus s.l. & $0(62)$ & $0(62)$ & $0(41)$ \\
\hline & An. pampanai & $0(5)$ & $0(5)$ & $0(5)$ \\
\hline
\end{tabular}

${ }^{1}$ All ELISA positive specimens were molecular identified as An. dirus s.s.

${ }^{2}$ All ELISA positive specimens were molecular identified as An. sawadwongporni

${ }^{3}$ The ELISA positive specimen was molecular identified as An. minimus s.s.

${ }^{4}$ The identification of the ELISA positive specimen was molecular confirmed as An. pampanai 
respectively), in the evening their use decreased to $54 \%$ in the villages and to only $21 \%$ at the forest plot, and at night they were hardly used (Peeters Grietens personal comment). Despite the low use of the LLIH during the night, the community based-cluster randomized trial showed an impact, mainly in the high risk group [8]. In Vietnam the risk of being bitten by a Plasmodium infectious mosquito is, as shown by this study, mainly during the early evening. Intervention, such as LLIH, that protects people during this period, when treated bed nets are not used, should be beneficial. Indeed, in Cambodia it has been shown that persons sitting in a LLIH are less bitten than persons sitting in a control hammock, though the personal protective effect of LLIH was variable according to mosquito species, villages and surveys [7]. The current study, suggested that no overall impact existed on the Anopheles density in forest, an expected result since the LLIH coverage was likely too low and too scattered to have any impact. Even if LLIH are not inducing full protection against the bites of all vectors, they may contribute protecting people. Insecticide-treated nets are however still needed as mosquitoes were active during the entire night and transmission also occurs during that period. Moreover, people are also exposed to mosquito bites when going to and from the forest plot hut in the early morning or in the evening. Elimination of malaria in this setting has to take this complexity into account and must rely on different control measures and personal protection methods.

Plasmodium infected mosquitoes were only detected in the forest and on the way from the village to the forest. This does not imply that malaria transmission is absent in the villages [5], but that the level of transmission was below the detection limits of the applied screening technique. As vector density was low inside the villages, fewer mosquitoes were assayed, decreasing the chance of sporozoite detection. Moreover, in areas of low transmission other techniques than estimating the entomological inoculation rate are needed to more accurately assess the trends in transmission. This can be done by serological test measuring the force of infection [25]. However, the CSP-ELISA technique followed by a PCR confirmation is still needed to identify Anopheles species bearing Plasmodium sporozoites. In Ninh Thuan province 24 different Anopheles species were identified among which four were incriminated as vectors of malaria, namely An. dirus s.s., An. sawadwogporni, An. minimus s.s. and An. pampanai. The contribution of the different Anopheles species to the transmission of the two Plasmodium species seemed to be different, though the numbers were too low to support this statistically. Anopheles dirus was the main vector for P. falciparum whereas An. sawadwongporni played an important role as $P$. vivax vector. In areas where $P$. falciparum and
$P$. vivax co-occur, successful control leads often to a shift from $P$. falciparum to P. vivax [26-28]. The elimination of $P$. falciparum may even reveal $P$. vivax, previously hidden in mixed infections [29]. As the extrinsic incubation period of $P$. vivax is shorter, other Anopheles species, often considered as secondary vectors, may become important in malaria transmission in Ninh Thuan Province. This may change the epidemiology of the disease and make vector control more complex. Anopheles sawadwongporni for example showed to be a very early biter and was still active after $6 \mathrm{am}$. It was also the most abundant species in both the forest and the village.

\section{Conclusion}

Vector control tools need to be adapted to the local context taking into account human and vector behaviour. A thorough knowledge of the biology, ecology and behaviour of vector species is essential to understand malaria transmission and to design appropriate vector control strategies. In Southeast Asia malaria is becoming more and more restricted to specific areas where human and vector characteristics alter the known features of malaria epidemiology, requiring alternative or adapted control strategies. This study showed clearly that risk of malaria transmission in the malaria foci is spread over the entire night, from dusk to dawn, requiring a combination of complementary vector control measures, such as LLIH and LN that can be used during different periods of the night. Moreover, with the progressive elimination of malaria in Southeast Asia, it can be expected that $P$. vivax becomes more prominent. Transmission of this Plasmodium species is not only assured by the main vectors but also by less known vectors as shown in this study. This poses new challenges when changing objectives from control to elimination of malaria from Southeast Asia and the need to focus not only on the so-called main vector species.

\section{Acknowledgements}

We are grateful to the staff of the Centre for Malariology, Parasitology \& Entomology of Ninh Thuan province, the health staff of the districts and communes who were involved in the planning and implementation of the study. We acknowledge the inhabitants of the study villages who supported the study. This work was carried out in the framework of the study on LLIH efficacy supported by the UBS-Opitmus Foundation, Zurich (Switzerland). The current study was financially supported by the Institutional Collaboration between NIMPE and the Institute of Tropical Medicine, Belgium, supported by the Belgian Co-operation (Directorate-General for Development Co-operation).

\section{Author details}

${ }^{1}$ Institute of Tropical Medicine Antwerp, Dept. Parasitology, Nationalestraat 155, B-2000 Antwerpen, Belgium. ${ }^{2}$ National Institute of Malariology, Parasitology and Entomology, Dept. Entomology, Luong The Vinh street, B.C. 10.200 Tu Liem, Hanoi, Vietnam. ${ }^{3}$ Centre for Malariology, Parasitology \& Entomology of Ninh Thuan province, Vietnam. ${ }^{4}$ Institute of Tropical Medicine, Dept. Animal Health, Nationalestraat 155, B-2000 Antwerpen, 
Belgium. Institute of Health and Society, Université Catholique de Louvain, Brussels, Belgium. ${ }^{6}$ Department of Biomedical Sciences, Faculty of Pharmaceutical, Veterinary and Biomedical Sciences, University of Antwerp, Universiteitsplein 1, B-2610 Antwerpen, Belgium.

\section{Authors' contributions}

WVB, MC, and HDT designed the study. WVB supervised the work critically at all stages, carried out the data analysis and drafted the manuscript. HDT, LXH, NVH, NVC and NDL facilitated and carried out the field work. LD, PR carried out the ELISA assays and molecular identification of the collected mosquitoes and critically reviewed the manuscript. NS contributed to the statistical analysis and critically reviewed the manuscript. MC and UD critically reviewed the manuscript. All authors read and approved the final manuscript.

\section{Competing interests}

The authors declare that they have no competing interests

Received: 20 July 2010 Accepted: 23 December 2010 Published: 23 December 2010

\section{References}

1. Verlé $\mathrm{P}$, Tuy TQ, Lieu TT, Kongs A, Coosemans M: New challenges for malaria control in northern Vietnam. Res Rev Parasitol 1998, 58:169-174

2. Barat LM: Four malaria success stories: How malaria burden was successfully reduced in Brazil, Eritrea, India, and Vietnam. Am J Trop Med Hyg 2006, 74:12-16.

3. Erhart A: Malaria control in Vietnam: successes and challenges University of Antwerp; 2006.

4. Erhart A, Thang ND, Hung NQ, Toi LV, Hung LX, Tuy TQ, Cong LD, Speybroeck N, Coosemans M, D'alessandro U: Forest malaria in Vietnam: A challenge for control. Am J Trop Med Hyg 2004, 70:110-118.

5. Erhart A, Thang ND, Ky PV, Tinh TT, Van Overmeir C, Speybroeck N, Obsomer V, Hung LX, Thuan LK, Coosemans M, D'alessandro U: Epidemiology of forest malaria in central Vietnam: a large scale crosssectional survey. Malar J 2005, 4:58.

6. Trung HD, Van Bortel W, Sochantha T, Keokenchanh K, Briët OJT, Coosemans M: Behavioural heterogeneity of Anopheles species in ecologically different localities in Southeast Asia: a challenge for vector control. Trop Med Int Health 2005, 10:251-262.

7. Sochantha T, Van Bortel W, Savonnaroth S, Marcotty T, Speybroeck N, Coosemans M: Personal protection by long-lasting insecticidal hammocks against the bites of forest malaria vectors. Trop Med Int Health 2010, 15:336-341.

8. Thang ND, Erhart A, Speybroeck N, Nguyen XX, Nguyen NT, Pham VK, Le XH, Le KT, Coosemans M, D'alessandro U: Long-lasting insecticidal hammocks for controlling forest malaria: a community-based trial in a rural area of Central Vietnam. PLOS ONE 2009, 4:e7369.

9. Grietens KP, Xa NX, Van Bortel W, Thang ND, Ribera JM, Truong BN, Ky PV, Hung LX, D'alessandro U, Erhart A: Low perception of malaria risk among the Ra-glai ethnic minority in south-central Vietnam: implications for forest malaria control. Malar J 2010, 9:23.

10. IMPE: Keys to the Anopheles in Vietnam (Adults-Pupae-Larvae) (In Vietnamese) Hanoi: Department of Entomology, Institute of Malariology, Parasitology, and Entomology; 1987.

11. Burkot TR, Zavala F, Gwadz RW, Collins FH, Nussenzweig RS, Roberts DR: Identification of malaria-infected mosquitoes by a two-site enzymelinked immunosorbent assay. Am J Trop Med Hyg 1984, 33:227-231.

12. Wirtz RA, Burkot TR, André RG, Rosenberg R, Collins WE, Roberts DR: Indentification of Plasmodium vivax sporozoites in mosquitoes using an enzyme linked immunosorbent assay. Am J Trop Med Hyg 1985, 34:1048-1054.

13. Wirtz RA, Sattabongkot J, Hall T, Burkot TR, Rosenberg R: Development and evaluation of an enzyme-linked immunosorbent assay for Plasmodium vivax-VK247 sporozoites. J Med Entomol 1992, 29:854-857.

14. Mangold KA, Manson RU, Koay ESC, Stephens L, Regner M, Thomson RB, Peterson LR, Kaul KL: Real-time PCR for detection and identification of Plasmodium spp. J Clin Microbiol 2005, 43:2435-2440

15. Van Bortel W, Trung HD, Roelants P, Harbach RE, Backeljau T, Coosemans M: Molecular identification of Anopheles minimus s.l. beyond distinguishing the members of the species complex. Insect Mol Biol 2000, 9:335-340.
16. Walton C, Handley JM, Kuvangkadilok C, Collins FH, Harbach RE, Baimai V, Bultin RK: Identification of five species of the Anopheles dirus complex from Thailand, using allele-specific polymerase chain reaction. Med Vet Entomol 1999, 13:24-32.

17. Beebe NW, Saul A: Discrimination of all members of the Anopheles punctulatus complex by polymerase chain reaction-restriction fragment length polymorphism analysis. Am J Trop Med Hyg 1995, 53:478-481.

18. Walton C, Somboon P, O'Loughlin SM, Zhang S, Harbach RE, Linton YM, Chen B, Nolan K, Duong S, Fong MY, Vythilingum I, Mohammed ZD, Trung HD, Butlin RK: Genetic diversity and molecular identification of mosquito species in the Anopheles maculatus group using the ITS2 region of rDNA. Infect Genet Evol 2007, 7:93-102.

19. Hair JF, Anderson RE, Tatham RL, Black WC: Multivariate Data Analysis. 5 edition. New Jersey: Prentivce-Hall International, Inc; 1998.

20. Obsomer V, Defourny P, Coosemans M: The Anopheles dirus complex: spatial distribution and environmental drivers. Malar J 2007, 6:26.

21. Beier JC, Killeen GF, Githure Jl: Short report: Entomologic inoculation rates and Plasmodium falciparum malaria prevalence in Africa. Am J Trop Med Hyg 1999, 61:109-113.

22. Okello PE, Van Bortel W, Byaruhanga AM, Correwyn A, Roelants $P$, Talisuna A, D'alessandro U, Coosemans M: Variation in malaria transmission intensity in seven sites throughout Uganda. Am J Trop Med Hyg 2006, 75:219-225.

23. Shaukat AM, Breman JG, McKenzie FE: Using the entomological inoculation rate to assess the impact of vector control on malaria parasite transmission and elimination. Malaria J 2010, 9:122.

24. Trung HD, Van Bortel W, Sochanta T, Keokenchanh K, Quang NT, Cong LD, Coosemans M: Malaria transmission and major malaria vectors in different geografical areas of Southeast Asia. Trop Med Int Health 2004, 9:230-237

25. Cook J, Reid H, lavro J, Kuwahata M, Taleo G, Clements A, McCarthy J, Vallely A, Drakeley C: Using serological measures to monitor changes in malaria transmission in Vanuatu. Malar J 2010, 9:169.

26. Konchom S, Singhasivanon P, Kaewkungwal J, Chupraphawan S, Thimasarn K, Kidson C, Rojanawatsirivet C, Yimsamran S, Looareesuwan S: Trend of malaria incidence in highly endemic provinces along the Thai borders, 1991-2001. Southeast Asian J Trop Med Public Health 2003, 34:486-494.

27. Sattabongkot J, Tsuboi T, Zollner GE, Sirichaisinthop J, Cui LW: Plasmodium vivax transmission: chances for control? Trends Parasitol 2004, 20:192-198.

28. Oliveira-Ferreira J, Lacerda MV, Brasil P, Ladislau JL, Tauil PL, DanielRibeiro CT: Malaria in Brazil: an overview. Malar J 2010, 9:115.

29. Mayxay M, Pukrittayakamee S, Newton PN, White NJ: Mixed-species malaria infections in humans. Trends Parasitol 2004, 20:233-240.

30. Perneger TV: What's wrong with Bonferroni adjustments. Br Med J 1998, 316:1236-1238.

doi:10.1186/1475-2875-9-373

Cite this article as: Van Bortel et al:: Malaria transmission and vector behaviour in a forested malaria focus in central Vietnam and the implications for vector control. Malaria Journal 2010 9:373.

\section{Submit your next manuscript to BioMed Central and take full advantage of:}

- Convenient online submission

- Thorough peer review

- No space constraints or color figure charges

- Immediate publication on acceptance

- Inclusion in PubMed, CAS, Scopus and Google Scholar

- Research which is freely available for redistribution

Submit your manuscript at www.biomedcentral.com/submit
C Biomed Central 